ITP-UU-05/33

SPIN-05/27

August 2005

\title{
Supersymmetric Black Holes ${ }^{1}$
}

\author{
Bernard de Wit \\ Institute for Theoretical Physics \& Spinoza Institute, \\ Utrecht University, The Netherlands \\ b.dewit@phys.uu.nl
}

\begin{abstract}
Recent insights from string theory and supergravity on the macroscopic and the microscopic description of black hole entropy are discussed.
\end{abstract}

\footnotetext{
${ }^{1}$ To be published in the proceedings of the Symposium in honor of the 70th birthday of Julius Wess, München, 10-11 January, 2005.
} 


\section{Introduction}

It is a great pleasure and an honor to be a speaker at this symposium on the occasion of Julius Wess' seventieth birthday. Julius' scientific achievements are impressive and his contributions to supersymmetry have inspired the scientific activities of many of us during a considerable part of our careers. In addition to his research Julius has served the physics community in many other capacities. Few physicists of his stature have had such an impact on the structuring of science and scientific education in and outside Germany. Here I am also thinking of his more recent Balkan initiative to rebuild the science infrastructure in former Yugoslavia after the disruptive and painful events in the nineties.

On this occasion I would like to review certain aspects of the relation between the macroscopic and microscopic descriptions of black holes. Black holes are, roughly speaking, solutions of Einstein's equations of general relativity that exhibit an event horizon. From inside this horizon, nothing (and in particular, no light) can escape. In the context of this talk, it suffices to think of spherically symmetric and static black holes, with a flat space-time geometry at spatial infinity. By definition, the region inside the horizon is not in the backward lightcone of future timelike infinity. However, since the discovery of Hawking radiation it has become clear that many of the above classical features of black holes are modified.

I will be considering black holes in four space-time dimensions, carrying electric and/or magnetic charges. Such solutions can be described by Einstein-Maxwell theory, the classical field theory for gravity and electromagnetism. The most general static black holes of this type correspond to the Reissner-Nordstrom solutions, characterized by a charge $Q$ and a mass $M$. In the presence of magnetic charges, $Q$ is replaced by $\sqrt{q^{2}+p^{2}}$ in most formulae, where $q$ and $p$ denote the electric and the magnetic charge, respectively. For zero charges one is dealing with Schwarzschild black holes. Two quantities associated with the black hole horizon are the area $A$ and the surface gravity $\kappa_{\mathrm{s}}$. The area is simply the area of the two-sphere defined by the horizon. The surface gravity, which is constant on the horizon, is related to the force (measured at spatial infinity) that holds a unit test mass in place. The mass $M$ and charge $Q$ of the black hole are not directly associated with the horizon and can be expressed by appropriate surface integrals at spatial infinity.

As is well known, there exists a striking correspondence between the laws of thermodynamics and the laws of black hole mechanics [1]. Of particular importance is the first law, which, for thermodynamics, states that the variation of the total energy is equal to the temperature times the variation of the entropy, modulo work terms, for instance proportional to a change of the volume. The corresponding formula for black holes expresses how the variation of the black hole mass is related to the variation of the horizon area, up to work terms proportional to the variation of the angular momentum. In addition there can also be a term proportional to a variation of the charge, multiplied by the electric/magnetic potential $\mu$ at the horizon. Specifically, the first law of thermodynamics, $\delta E=T \delta S-p \delta V$, translates into

$$
\delta M=\frac{\kappa_{\mathrm{S}}}{2 \pi} \frac{\delta A}{4}+\mu \delta Q+\Omega \delta J
$$


The reason for factorizing the first term on the right-hand side in this particular form, is that $\kappa_{\mathrm{S}} / 2 \pi$ represents precisely the Hawking temperature [2]. This then leads to the identification of the black hole entropy in terms of the horizon area,

$$
\mathcal{S}_{\text {macro }}=\frac{1}{4} A
$$

a result that is known as the area law [3. In these equations the various quantities have been defined in Planck units, meaning that they have been made dimensionless by multiplication with an appropriate power of Newton's constant (we will set $\hbar=c=1$ ). This constant appears in the Einstein-Hilbert Lagrangian according to $\mathcal{L}_{\mathrm{EH}}=-\left(16 \pi G_{\mathrm{N}}\right)^{-1} \sqrt{|g|} R$. With this normalization the quantities appearing in the first law are independent of the scale of the metric.

Einstein-Maxwell theory can be naturally embedded into $N=2$ supergravity which may lead to an extension with a variety of abelian gauge fields and a related number of massless scalar fields (often called 'moduli' fields, for reasons that will become clear later on). At spatial infinity these moduli fields will tend to a constant, and the black hole mass will depend on these constants, thus introducing additional terms on the right-hand side of (1).

For Schwarzschild black holes the only relevant parameter is the mass $M$ and I note the relations,

$$
A=16 \pi M^{2}, \quad \kappa_{\mathrm{s}}=\frac{1}{4 M},
$$

consistent with (11). For the Reissner-Nordstrom black hole, the situation is more subtle. Here one distinguishes three different cases. For $M>Q$ one has the non-extremal solutions, which exhibit two horizons, an exterior event horizon and an interior horizon. When $M=Q$ one is dealing with an extremal black hole, for which the two horizons coincide and the surface gravity vanishes. In that case one has

$$
A=4 \pi M^{2}, \quad \kappa_{\mathrm{s}}=0, \quad \mu=Q \sqrt{\frac{4 \pi}{A}} .
$$

It is straightforward to verify that this result is consistent with (11) for variations in the subspace of extremal black holes (i.e., with $\delta M=\delta Q$ ). Because the surface gravity vanishes, one might expect the entropy to vanish as suggested by the third law of thermodynamics. Obviously, that is not the case as the horizon area remains finite for zero surface gravity. Finally, solutions with $M<Q$ are not regarded as physically acceptable. Their total energy is less than the electromagnetic energy alone and they no longer have an event horizon but exhibit a naked singularity. Hence extremal black holes saturate the bound $M \geq Q$ for physically acceptable black hole solutions.

When embedding Einstein-Maxwell theory into a complete supergravity theory, the above classification has an interpretation in terms of the supersymmetry algebra. This algebra has a central extension proportional to the black hole charge(s). Unitary representations of the supersymmetry algebra must necessarily have masses that are larger than or equal to the charge. When this bound is saturated, one is dealing with so-called BPS supermultiplets. Such supermultiplets are smaller than the generic massive $N=2$ supermultiplets and have 
a different spin content. Because of this, BPS states are stable under (adiabatic) changes of the coupling constants, and the relation between charge and mass remains preserved. This important feature of BPS states will be relevant for what follows.

\section{On macroscopic and microscopic descriptions}

A central question in black hole physics concerns the statistical interpretation of the black hole entropy. String theory has provided new insights here 4], which have led to important results. In this context it is relevant that strings live in more that four space-time dimensions. In most situations the extra dimensions are compactified on some internal manifold $X$ and one is dealing with the usual Kaluza-Klein scenario leading to effective field theories in four dimensions, describing low-mass modes of the fields associated with certain eigenfunctions on the internal manifold.

Hence the original space-time will locally be a product $M^{4} \times X$, where $M^{4}$ denotes the four-dimensional space-time that one experiences in daily life. I will denote the coordinates of $M^{4}$ by $x^{\mu}$ and those of $X$ by $y^{m}$. In the situation described above there exists a corresponding space $X$ at every point $x^{\mu}$ of $M^{4}$, whose size is such that it will not be directly observable. However, this space $X$ does not have to be the same at every point in $M^{4}$, and moving through $M^{4}$ one may encounter various spaces $X$ which may or may not be equivalent. Usually these spaces belong to some well-defined class of fixed topology parametrized by certain moduli. These moduli will appear as fields in the four-dimensional effective field theory. For instance, suppose that the spaces $X$ are $n$-dimensional tori $T^{n}$. The metric of $T^{n}$ will appear as a field in the four-dimensional theory and is related to the torus moduli. Hence, when dealing with a solution of the four-dimensional theory that is not constant in $M^{4}$, each patch in $M^{4}$ has a nontrivial image in the space of moduli that parametrize the internal spaces $X$.

For a black hole solution, viewed in this higher-dimensional perspective, the fields, and in particular the four-dimensional space-time metric, will vary nontrivially over $M^{4}$, and so will the internal space $X$. When moving to the center of the black hole the gravitational fields will become strong and the local product structure into $M^{4} \times X$ could break down. One feature of string theory is, however, absent in this field-theoretic approach which captures the local degrees of freedom of strings and branes: extended objects carry also global degrees of freedom as they can wrap themselves around nontrivial cycles of the internal space $X$. This wrapping tends to take place at a particular position in $M^{4}$ so in the context of the fourdimensional effective field theory, this will reflect itself as a pointlike object. This wrapped object is the string theory representation of the black hole!

One is thus dealing with two complementary pictures of the black hole. One based on general relativity where a point mass generates a global solution of space-time with strongly varying gravitational fields, which I shall refer to as the macroscopic description. The other one, based on the internal space where an extended object is entangled in one of its cycles, does not involve gravitational fields and can easily be described in flat space-time. This description will be refered to as microscopic. To understand how these two descriptions are 
related is far from easy, but a connection must exist in view of the fact that gravitons are closed string states which interact with the wrapped branes. These interactions are governed by the string coupling constant $g_{\mathrm{s}}$ and one is thus confronted with an interpolation in that coupling constant. In principle, such an interpolation is very difficult to carry out, so that a realistic comparison between microscopic and macroscopic results is usually impossible. However, reliable predictions are possible for extremal black holes! In a supersymmetric setting extremal black holes are BPS and, as I indicated earlier, in that situation there are reasons to trust such interpolations. Indeed, it has be shown that the predictions based on these two alternative descriptions can be successfully compared and new insights about black holes can be obtained.

But how do the wrapped strings and branes represent themselves in the effective action description and what governs their interactions with the low-mass fields? Here it is important to realize that the massless four-dimensional fields are associated with harmonic forms on $X$. Harmonic forms are in one-to-one correspondence with so-called cohomology groups consisting of equivalence classes of forms that are closed but not exact. The number of independent harmonic forms of a given degree is given by the so-called Betti numbers, which are fixed by the topology of the spaces $X$. When expanding fields in a Kaluza-Klein scenario, the number of corresponding massless fields can be deduced from an expansion in terms of tensors on $X$ corresponding to the various harmonic forms. The higher-dimensional fields $\Phi(x, y)$ thus decompose into the massless fields $\phi^{A}(x)$ according to (schematically),

$$
\Phi(x, y)=\phi^{A}(x) \omega_{A}(y)
$$

where $\omega_{A}(y)$ denotes the independent harmonic forms on $X$. The above expression, when substituted into the action of the higher-dimensional theory, leads to interactions of the fields $\phi^{A}$ proportional to the 'coupling constants',

$$
C_{A B C \cdots} \propto \int_{X} \omega_{A} \wedge \omega_{B} \wedge \omega_{C} \cdots
$$

These constants are known as intersection numbers, for reasons that will become clear shortly.

I already mentioned that the Betti numbers depend on the topology of $X$. This is related to Poincaré duality, according to which cohomology classes are related to homology classes. The latter consist of submanifolds of $X$ without boundary that are themselves not a boundary of some other submanifold of $X$. This is precisely relevant for wrapped branes which indeed cover submanifolds of $X$, but are not themselves the boundary of a submanifold because otherwise the brane could collapse to a point. Without going into detail, this implies that there exists a dual relationship between harmonic $p$-forms $\omega$ and $\left(d_{X}-p\right)$-cycles, where $d_{X}$ denotes the dimension of $X$. One can therefore choose a homology basis for the $\left(d_{X}-p\right)$ cycles dual to the basis adopted for the $p$-forms. Denoting this basis by $\Omega_{A}$ the wrapping of an extended object can now be characterized by specifying its corresponding cycle $\mathcal{P}$ in terms of the homology basis,

$$
\mathcal{P}=p^{A} \Omega_{A} .
$$


The integers $p^{A}$ count how many times the extended object is wrapped around the corresponding cycle, so one is actually dealing with integer-valued cohomology and homology. The wrapping numbers $p^{A}$ reflect themselves as magnetic charges in the effective action. The electric charges are already an integer part of the effective action, because they are associated with gauge transformations that usually originate from the higher-dimensional theory.

Owing to Poincaré duality it is thus very natural that the winding numbers interact with the massless modes in the form of magnetic charges, so that they can be incorporated in the effective action. Before closing this section, I note that, by Poincaré duality, one can express the number of intersections by

$$
\mathcal{P} \cdot \mathcal{P} \cdot \mathcal{P} \cdots=C_{A B C \cdots} p^{A} p^{B} p^{C} \cdots
$$

This is a topological characterization of the wrapping, which will appear in later formulae.

\section{$3 \quad$ Black holes in M/String Theory}

As an example I now discuss the black hole entropy derived from both microscopic and macroscopic arguments in a special case. Consider M-theory, which, in the strong coupling limit of type-IIA string theory, is described by eleven-dimensional supergravity. The latter is invariant under 32 supersymmetries. Seven of the eleven space-time dimensions are compactified on an internal space which is the product of a Calabi-Yau threefold (a three-dimensional complex manifold, which henceforth I denote by $C Y_{3}$ ) times a circle $S^{1}$. Such a space breaks part of the supersymmetries and only 8 of them are left unaffected. In the context of the four-dimensional space-time $M^{4}$, these 8 supersymmetries are encoded into two independent Lorentz spinors and for that reason this symmetry is referred to as $N=2$ supersymmetry. Hence the effective four-dimensional field theory will be some version of $N=2$ supergravity.

M-theory contains a five-brane and this is the microscopic object that is responsible for the black holes that I consider; the five-brane has wrapped itself on a 4-cycle $\mathcal{P}$ of the $C Y_{3}$ space [5]. Alternatively one may consider this class of black holes in type-IIA string theory, with a 4 -brane wrapping the 4 -cycle 6 . The 4-cycle is subject to certain requirements which will be mentioned in due course.

The massless modes captured by the effective field theory correspond to harmonic forms on the $C Y_{3}$ space; they do not depend on the $S^{1}$ coordinate. The 2 -forms are of particular interest. In the effective theory they give rise to vector gauge fields $A_{\mu}{ }^{A}$, which originate from the rank-three tensor gauge field in eleven dimensions. In addition there is an extra vector field $A_{\mu}{ }^{0}$ coresponding to a 0 -form which is related to the graviphoton associated with $S^{1}$. This field will couple to the electric charge $q_{0}$ associated with momentum modes on $S^{1}$ in the standard Kaluza-Klein fashion. The 2-forms are dual to 4-cyles and the wrapping of the five-brane is encoded in terms of the wrapping numbers $p^{A}$, which appear in the effective field theory as magnetic charges which couple to the gauge fields $A_{\mu}{ }^{A}$. Here one sees Poincaré duality at work, as the magnetic charges couple nicely to the corresponding gauge fields. For a Calabi-Yau three-fold, there is a triple intersection number $C_{A B C}$ which appears in the 
three-point couplings of the effective field theory. There is a subtle topological feature that I have not explained before, which is typical for complex manifolds containing 4-cycles, namely the existence of another quantity of topological interest known as the second Chern class. The second Chern class is a 4-form whose integral over a four-dimensional Euclidean space defines the instanton number. The 4 -form can be integrated over the 4 -cycle $\mathcal{P}$ and yields $c_{2 A} p^{A}$, where the $c_{2 A}$ are integers.

Let me now turn to the microscopic counting of degrees of freedom [5]. These degrees of freedom are associated with the massless excitations of the wrapped five-brane characterized by the wrapping numbers $p^{A}$ on the 4 -cycle. The 4-cycle $\mathcal{P}$ must correspond to a holomorphically embedded complex submanifold in order to preserve 4 supersymmetries. The massless excitations of the five-brane are then described by a $(1+1)$-dimensional superconformal field theory (the reader may also consult [7]). Because I have compactified the spatial dimension on $S^{1}$, one is dealing with a closed string with left- and right-moving states. The 4 supersymmetries of the conformal field theory reside in one of these two sectors, say the right-handed one. Conformal theories in $1+1$ dimensions are characterized by a central charge, and in this case there is a central charge for the right- and for the left-moving sector separately. These central charges are expressible in terms of the wrapping numbers $p^{A}$ and depend on the intersection numbers and the second Chern class, according to

$$
\begin{aligned}
c_{L} & =C_{A B C} p^{A} p^{B} p^{C}+c_{2 A} p^{A}, \\
c_{R} & =C_{A B C} p^{A} p^{B} p^{C}+\frac{1}{2} c_{2 A} p^{A} .
\end{aligned}
$$

I should stress that the above result is far from obvious and holds only under the condition that the $p^{A}$ are large. In that case every generic deformation of $\mathcal{P}$ will be smooth. Under these circumstances it is possible to relate the topological properties of the 4-cycle to the topological data of the Calabi-Yau space.

One can now choose a state of given momentum $q_{0}$ which is supersymmetric in the rightmoving sector. From rather general arguments it follows that such states exist. The corresponding states in the left-moving sector have no bearing on the supersymmetry and these states have a certain degeneracy depending on the value of $q_{0}$. In this way one has a tower of BPS states invariant under 4 supersymmetries, built on supersymmetric states in the rightmoving sector and comprising corresponding degenerate states in the left-moving sector. One can then use Cardy's formula, which states that the degeneracy of states for fixed but large momentum (large as compared to $c_{L}$ ) equals $\exp \left[2 \pi \sqrt{\left|q_{0}\right| c_{L} / 6}\right]$. This leads to the following expression for the entropy,

$$
\mathcal{S}_{\text {micro }}(p, q)=2 \pi \sqrt{\frac{1}{6}\left|\hat{q}_{0}\right|\left(C_{A B C} p^{A} p^{B} p^{C}+c_{2 A} p^{A}\right)},
$$

where $q_{0}$ has been shifted according to $\hat{q}_{0}=q_{0}+\frac{1}{2} C^{A B} q_{A} q_{B}$, with $C^{A B}$ the inverse of $C_{A B}=C_{A B C} p^{C}$. This modification is related to the fact that the electric charges associated with the gauge fields $A_{\mu}{ }^{A}$ will interact with the M-theory two-brane [5]. The existence of this interaction can be inferred from the fact that the two-brane interacts with the rank-three tensor field in eleven dimensions, from which the vector gauge fields $A_{\mu}{ }^{A}$ originate. 
I stress that the above results apply in the case of large charges. The first term proportional to the triple intersection number is obviously the leading contribution whereas the terms proportional to the second Chern class are subleading. The importance of the subleading terms will become more clear in later sections. Having obtained a microscopic representation of a BPS black hole, it now remains to make contact with it by deriving the corresponding black hole solution directly in the $N=2$ supergravity theory. This is discussed below.

\section{An entropy formula for $N=2$ supergravity}

The charged black hole solutions in $N=2$ supergravity are invariant under 4 of the 8 supersymmetries. They are solitonic, and interpolate between fully supersymmetric configurations at the horizon and at spatial infinity. At spatial infinity, where the effect of the charges can be ignored, one has flat Minkowski space-time. The scalar moduli fields tend to certain (arbitrary) values on which the black hole mass will depend. At the horizon the situation is rather different, because here the charges are felt and one is not longer dealing with flat space-time, but with a so-called Bertotti-Robinson space, $\mathrm{AdS}_{2} \times S^{2}$. In that situation the requirement of full $N=2$ supersymmetry is highly restrictive and for spherical geometries one can prove that the values of the moduli fields at the horizon are in fact fixed in terms of the charges. The corresponding equations are known as the attractor equations [8, 9, 10] and they apply quite generally. For effective actions with interactions quadratic in the curvature the validity of these attractor equations was established in [11.

The attractor equations play a crucial role as they ensure that the entropy, a quantity that is associated with the horizon, will depend on the black hole charges and not on other quantities, in line with the microscopic results presented in the previous section. Hence one is interested in studying charged black hole solutions which are BPS, meaning that they are invariant under 4 supersymmetries. The matter supermultiplets contain the gauge fields $A_{\mu}{ }^{A}$ coupling to electric and magnetic charges $q_{A}$ and $p^{A}$, respectively. In addition there is one extra graviphoton field $A_{\mu}{ }^{0}$ which may couple to charges $q_{0}$ and $p^{0}$. When comparing to the solutions of the previous section, one obviously sets $p^{0}=0$, although from the supergravity point of view there is no need for such a restriction.

However, there is an infinite variety of $N=2$ supergravity actions coupling to vector multiplets. Fortunately these actions can be conveniently encoded into holomorphic functions that are homeogeneous of second degree [12. In the case at hand the simplest action is, for instance, based on the function,

$$
F(Y)=-\frac{1}{6} \frac{C_{A B C} Y^{A} Y^{B} Y^{C}}{Y^{0}},
$$

where the holomophic variables $Y^{I}(I=0, A)$ are associated with the vector multiplets; they can be identified projectively with the scalar moduli fields that are related to a subset of the moduli of the Calabi-Yau space. The black hole solution will thus encode the changes in the Calabi-Yau manifold when moving from the black hole horizon towards spatial infinity, 
precisely as discussed in a more general context in section 2. Note the presence of the triple intersection form $C_{A B C}$ which will appear in the interaction vertices of the corresponding Lagrangian.

The attractor equations also involve the function $F(Y)$. In terms of the quantities $Y^{I}$ and the first derivatives of $F(Y)$, they take the form,

$$
Y^{I}-\bar{Y}^{I}=\mathrm{i} p^{I}, \quad F_{I}(Y)-\bar{F}_{I}(\bar{Y})=\mathrm{i} q_{I}
$$

where $F_{I}(Y)=\partial F(Y) / \partial Y^{I}$. In principle these equations yield the horizon values of the $Y^{I}$ in terms of the charges. Depending on the values of the charges and on the complexity of the function $F(Y)$, it may not be possible to write down solutions in closed form.

The action corresponding to (11) gives rise to a black hole solution with charges $p^{A}, q_{A}$ and $q_{0}$ (with $p^{0}=0$ ). Its area can be calculated and is equal to

$$
A(p, q)=8 \pi \sqrt{\frac{1}{6}\left|\hat{q}_{0}\right| C_{A B C} p^{A} p^{B} p^{C}} .
$$

Upon invoking the area law this result leads precisely to the first part of the microscopic entropy (10). One has thus reproduced the leading contributions to the entropy from supergravity.

How can one reproduce the subleading terms in view of the fact that these terms scale differently whereas the function $F(Y)$ and the attractor equations all seem to scale uniformly? To explain how this is resolved I must first spend a few words on the reason why the function $F(Y)$ was homogeneous in the first place. The covariant fields corresponding to a vector supermultiplets comprise a so-called restricted chiral multiplet, which can be assigned a unique (complex) scaling weight. The $Y^{I}$ are proportional to the lowest component of these multiplets, and can be assigned the same scaling weight. Any (holomorphic) function of these restricted multiplets will define a chiral superfield, whose chiral superspace integral will lead to a supersymmetric action. However, in order to be able to couple to supergravity, this function must be homogeneous of second degree [12. To deviate from this homogeneity pattern in the determination of the entropy, one needs to introduce a new type of chiral superfield whose value at the horizon will be fixed in a way that breaks the uniformity of the scaling. There exists such a multiplet. Namely, from the fields of (conformal) supergravity itself, one can again extract a restricted chiral multiplet, which in this case comprises the covariant quantities associated with the supergravity fields. This time the restricted chiral multiplet is not a scalar, but an auxiliary anti-selfdual tensor, and just as before it can be assigned a unique scaling weight. Its lowest component is an auxiliary field that is often called the graviphoton field strength, which is strictly speaking a misnomer because it never satisfies a Bianchi identity. It appears in the transformation rule of the gravitino fields and in simple Lagrangians its field equations express it in terms of moduli-dependent linear combinations of other vector field strengths. The square of this restricted tensor defines a complex scalar field which constitutes the lowest component of a chiral supermultiplet and which is proportional to a field I will denote by $\Upsilon$, such that the (complex) scaling weight that can be assigned 
to $\Upsilon$ is twice that of the $Y^{I}$. More general supergravity Lagrangians can then described by holomorphic functions $F(Y, \Upsilon)$ that are homogeneous of degree 2, i.e.,

$$
F\left(\lambda Y^{I}, \lambda^{2} \Upsilon\right)=\lambda^{2} F\left(Y^{I}, \Upsilon\right)
$$

A nontrivial dependence on $\Upsilon$ in the function $F(Y, \Upsilon)$ has important consequences, because the supermultiplet of which $\Upsilon$ is the first component contains other components with terms quadratic in the Riemann tensor. Hence actions based on a function (14) with a nontrivial dependence on $\Upsilon$ will contain terms proportional to the square of the Riemann tensor, multiplied by the first derivative of $F$ with respect to $\Upsilon$. The attractor equations (12) remain valid with $F(Y)$ replaced by $F(Y, \Upsilon)$. However, the field $\Upsilon$ has its own independent attractor value; at the horizon it must be equal to $\Upsilon=-64$, independent of the charges. This phenomenon explains why the area or the entropy formula are not necessarily a homogeneous function of the charges.

To fully reproduce the entropy formula (10) including the subleading terms proportional to the second Chern class, one may attempt a Lagrangian based on the function

$$
F(Y)=-\frac{1}{6} \frac{C_{A B C} Y^{A} Y^{B} Y^{C}}{Y^{0}}-\frac{c_{2 A}}{24 \cdot 64} \frac{Y^{A}}{Y^{0}} \Upsilon
$$

which is indeed holomorphic and homogeneous. On the basis of this modification one can again calculate the horizon area in the hope of recovering the entropy (13) upon use of the area law. However, the result is negative and it seems obvious that no solution can be found in this way 13 .

At this point the only way out is to no longer rely on the area law in extracting a value for the entropy. Indeed the area law is not expected to hold for actions that supersede the Einstein-Hilbert one. However, Wald has proposed an alternative definition of black hole entropy which can be used for any Lagrangian that is invariant under general coordinate transformations, and which is based on the existence of a conserved surface charge [14. The latter is related to the conventional Noether current associated with general coordinate transformations, which, for a gauge symmetry, can be written as a pure improvement term: the divergence of an antisymmetric tensor, called the Noether potential. It turns out that with the help of the Noether potential one can define a surface charge integrated over the boundary of a Cauchy surface, which for the black hole extends from spatial infinity down to the horizon. Changes in the continuous variety of black hole solutions should leave this charge unchanged. Under certain conditions one can show that the change of the surface integral at spatial infinity corresponds to the mass and angular momentum variations in the first law. Therefore one identifies the surface integral at the horizon with the entropy, so that the validity of the first law will remain ensured.

I should stress that there are various subtle points here, some of which have been discussed in [15]. The prescription based on the surface charge can be applied to standard Einstein gravity, in which case one just recovers the area law. But for theories with higher-derivatives, there are nontrivial correction terms, which follow from a calculation of the Noether potential. I should caution the reader that the relevant correction term in the case at hand does in fact 
not reside in the terms quadratic in the Riemann tensor, but in some other terms related to them by supersymmetry.

From an evaluation of the Noether potential, taking into account all the constraints imposed by the supersymmetry at the horizon, it follows that the entropy can be written in a universal form [16],

$$
\mathcal{S}_{\text {macro }}(p, q)=\pi\left[|Z|^{2}-265 \operatorname{Im} F_{\Upsilon}\right]_{\Upsilon=-64}
$$

Here the first term denotes the Bekenstein-Hawking entropy, because $|Z|^{2}=p^{I} F_{I}(Y, \Upsilon)-q_{I} Y^{I}$ is just the area in Planck units divided by $4 \pi$. This term is clearly affected by the presence of the higher-order derivative interactions. On top of that there is a second term proportional to the derivative of $F(Y, \Upsilon)$ with respect to $\Upsilon$. This term thus represents the deviation of the area law. The above formula applies to any $N=2$ supergravity solution.

Because of the fact that all quantities of interest are directly related to the holomorphic and homogeneous functions (14), the determination of the area and entropy is merely an algebraic exercise, which no longer requires to construct the full solution. Given the function $F(Y, \Upsilon)$ one first attempts to solve the attractor equations (12) with $F_{I}(Y)$ replaced by $F_{I}(Y, \Upsilon)$. Subsequently one determines the area and the entropy in terms of the charges. For the function (15) this was shown to lead precisely to the microscopic entropy formula (10). To exhibit the deviation from the area law, I also give the area (which is obviously not known from microscopic considerations),

$$
\frac{1}{4} A(p, q)=\frac{C_{A B C} p^{A} p^{B} p^{C}+\frac{1}{2} c_{2 A} p^{A}}{C_{A B C} p^{A} p^{B} p^{C}+c_{2 A} p^{A}} \mathcal{S}_{\text {macro }}(p, q) .
$$

Interestingly the proportionality factor is just the ratio $c_{R} / c_{L}$ of the two central charges defined in (9). By combining new ingredients from supergravity and general relativity it is thus possible to fully account for the black hole entropy that is obtained by counting microstates.

Before moving to the next section I wish to add some observations. In addition to being able to evaluate the properties of the black hole solution at the horizon one should also want to understand the full structure of the BPS black holes away from the horizon, in the presence of the interactions quadratic in the Riemann curvature. This was the subject of [11], where a rather general class of such solutions was studied, including multi-centered ones. I refer to that work for further details. It is also worth pointing out that sofar I have been basing myselves on the effective Wilsonian action. A priori, one does not expect that the final macroscopic description of black hole mechanics can be obtained within a Wilsonian framework.

\section{$5 \quad$ Heterotic black holes}

In [15, 17] the modified entropy formula (16) was applied to heterotic black holes. Although the formula is derived for $N=2$ supergravity, the result can readily be generalized to the 
case of heterotic $N=4$ supersymmetric compactifications. This involves an extension of the target-space duality group to $\mathrm{SO}(6,22)$ with a corresponding extension to 28 electric and 28 magnetic charges that take their values in a $\Gamma^{6,22}$ lattice. The $N=4$ supersymmetric heterotic models have dual realizations as type-II string compactifications on $K 3 \times T^{2}$. In contrast to $N=2$ Calabi-Yau compactifications, the holomorphic function which encodes the effective Wilsonian action is severely restricted in the $N=4$ case. Therefore it is often possible to obtain exact predictions in this context.

The relevant function for the heterotic case takes the following form in lowest order,

$$
F(Y)=-\frac{Y^{1} Y^{a} \eta_{a b} Y^{b}}{Y^{0}}
$$

where $a, b=2, \ldots, n$. This function will be modified in due course by a function of $\Upsilon$ and of the dilaton field $S=-\mathrm{i} Y^{1} / Y^{0}$. Let me first consider (18) in the absence of these modifications. Then the $2 n$ scalar moduli in the effective action are described by a nonlinear sigma model with the following target space,

$$
\mathcal{M}=\frac{\mathrm{SU}(1,1)}{\mathrm{U}(1)} \times \frac{\mathrm{SO}(2, n-1)}{\mathrm{SO}(2) \times \mathrm{SO}(n-1)} .
$$

The electric and magnetic charges transform under the action of the $\mathrm{SU}(1,1) \times \mathrm{SO}(2, n-1)$ isometry group. The first factor, $\mathrm{SU}(1,1)$, is associated with $S$-duality. This is a strongweak coupling duality which interchanges electric and magnetic charges. The second factor, $\mathrm{SO}(2, n-1)$, is associated with $T$-duality (also called target-space duality). There is a technical complication in the description based on (18) because the charges that follow from the $Y^{I}$ through the attractor equations are not in a convenient basis for $S$ - and $T$-duality. A proper basis is found upon interchanging the electric and magnetic charges $q_{1}$ and $p^{1}$ by an electric/magnetic duality. Fortunately there is no need to discuss this in any detail, as the entropy and area of the corresponding black holes depend only on $T$-duality invariants of the charges. Note that in the extension to $N=4$ the second factor of the target space (19) changes into $\mathrm{SO}(6,22) /[\mathrm{SO}(6) \times \mathrm{SO}(22)]$; this space is parametrized by the 132 scalar fields belonging to $22 N=4$ vector supermultiplets.

To be specific let me first present the result for the entropy and horizon area for the solution based on (18) as a function of the charges,

$$
\mathcal{S}_{\text {macro }}(p, q)=\frac{1}{4} A(p, q)=\pi \sqrt{q^{2} p^{2}-(p \cdot q)^{2}} .
$$

Here I used $T$-duality invariant combinations of the charges, defined by

$$
\begin{aligned}
q^{2} & =2 q_{0} p^{1}-\frac{1}{2} q_{a} \eta^{a b} q_{b}, \\
p^{2} & =-2 p^{0} q_{1}-2 p^{a} \eta_{a b} p^{b}, \\
p \cdot q & =q_{0} p^{0}-q_{1} p^{1}+q_{a} p^{a},
\end{aligned}
$$

where the $p^{I}$ and $q_{I}$ on the right-hand side are the charges that appear in the attractor equations (12) based on the function (18). While the combinations (21) are invariant under 
$T$-duality, they transform as an $\mathrm{SO}(2,1)$ vector under $S$-duality, such that the entropy formula (20) is invariant under both $T$ - and $S$-duality. The $S$-duality transformations, which constitute the group $\mathrm{SL}(2, \mathbb{Z})$, of the charges and of the dilaton field are related through the attractor equations.

In the $N=4$ theory, the charge lattice is $S$-duality invariant, meaning that the above transformations always leads to another point on the lattice that is physically realized. Note that the supergravity calculations yield no intrinsic definition of the normalization of the charge lattice and consequently the dilaton normalization is a priori not known. Hence the precise characterization of the arithmetic subgroup of $\mathrm{SL}(2)$ that defines the $S$-duality group is not obvious, but the crucial point is that the normalization of the dilaton is related to the normalization of the lattice of charges. Later on in this section I will relate the supergravity results to microscopic data which will confirm the above identifications. Observe that the invariants $p^{2}$ and $q^{2}$ are not positive definite. In fact in the limit of large charges they will both become negative.

When adding a function to (18) proportional to $\Upsilon$ and depending otherwise on the dilaton field $S$, it turns out that the target-space duality remains unaffected. However, $S$-duality is affected in general so the question is whether there exists a specific modification that leaves $S$-duality intact. This turns out to be the case, but one is forced to accept a certain amount of non-holomorphicity in the description [17. To derive this is rather nontrivial and I simply quote the result in a form that was indicated in [18,

$$
F(Y, \bar{Y}, \Upsilon, \bar{\Upsilon})=-\frac{Y^{1} Y^{a} \eta_{a b} Y^{b}}{Y^{0}}+\frac{\mathrm{i}}{64 \pi} \Upsilon \log \eta^{12}(S)+\frac{\mathrm{i}}{128 \pi}(\Upsilon+\bar{\Upsilon}) \log (S+\bar{S})^{6}
$$

where the non-holomorphic corrections reside in the last term. Here $\eta(S)$ denotes the Dedekind eta-function, which satisfies the asymptotic formula $\log \eta(S) \approx-\frac{1}{12} \pi S-\mathrm{e}^{-2 \pi S}+$ $\mathcal{O}\left(\mathrm{e}^{-4 \pi S}\right)$ for large positive real values of $S$. The presence of non-holomorphic terms in (22) is not entirely unexpected: the Wilsonian couplings are holomorphic but do not necessarily reflect the symmetries of the underlying theory, while the physical couplings must reflect the symmetry and may thus have different analyticity properties. The underlying reason is that the Wilsonian action is based on integrating out the massive modes above a certain scale; the effect of the massless modes must thus be included separately. The massless modes give rise to a certain non-local action, which is supersymmetric although it is not based on a chiral superspace density. Its explicit form has not been given so far, so that the non-holomorphic terms have been determined directly by requiring $S$-duality of the attractor equations and the entropy, and consistency with string perturbation theory. The results are in accord with the $N=4$ results of [19]. The normalization of the $R^{2}$-terms in the effective action has been fixed by using string-string duality, or, alternatively, by requiring agreement with the known asymptotic degeneracy of electrically charged black holes.

Including the non-holomorphic corrections, the result of [17] can be summarized as follows. The non-trivial attractor equations are the ones that determine the horizon value of the complex dilaton field $S$ in terms of the black hole charges. They read as follows (we have 
now set $\Upsilon$ to its horizon value),

$$
\begin{aligned}
|S|^{2} p^{2} & =q^{2}-\frac{2}{\pi}(S+\bar{S})\left(S \frac{\partial}{\partial S}+\bar{S} \frac{\partial}{\partial \bar{S}}\right) \log \left[(S+\bar{S})^{6}|\eta(S)|^{24}\right] \\
(S-\bar{S}) p^{2} & =-2 \mathrm{i} p \cdot q+\frac{2}{\pi}(S+\bar{S})\left(\frac{\partial}{\partial S}-\frac{\partial}{\partial \bar{S}}\right) \log \left[(S+\bar{S})^{6}|\eta(S)|^{24}\right] .
\end{aligned}
$$

The expression for the macroscopic entropy reads,

$$
\mathcal{S}_{\text {macro }}=-\pi\left[\frac{q^{2}-\mathrm{i} p \cdot q(S-\bar{S})+p^{2}|S|^{2}}{S+\bar{S}}\right]-2 \log \left[(S+\bar{S})^{6}|\eta(S)|^{24}\right],
$$

with the dilaton field subject to (23). The first term in this equation corresponds to one-fourth of the horizon area, which, via (23), is affected by the various corrections. The second term represents an extra modification, which explicitly contains the non-holomorphic correction. Both terms are invariant under target-space duality and $S$-duality. As explained above, $S$ duality was achieved at the price of including non-holomorphic terms, here residing in the $\log (S+\bar{S})$ terms.

In the $N=4$ setting one can distinguish two types of BPS-states. Purely electric or magnetic configurations constitute $1 / 2$-BPS states, whereas dyonic ones are 1/4-BPS states. For $N=2$ the distinction between the two types of states disappears and one has only $1 / 2$-BPS states. In the context of $N=4$, the generic BPS states are the dyonic ones, characterized by a nonzero value for $q^{2} p^{2}-(p \cdot q)^{2}$, while the $1 / 2$-BPS states are subject to the condition $q^{2} p^{2}-(p \cdot q)^{2}=0$. In the remainder of this section I restrict myself to the dyonic states. The macroscopic results given above can be confronted with an explicit formula for the microscopic degeneracy of BPS dyons in four-dimensional $N=4$ string theory proposed in [20. This proposal generalizes the expression for the degeneracies of electric heterotic string states (to be presented in the next section), to an expression that depends on both electric and magnetic charges such that it is formally covariant with respect to $S$-duality. In 20] it was already shown that the dyonic degeneracy was consistent with the area law, i.e. with (20) in the limit of large charges. Recently, this degeneracy was derived from the known degeneracies of black holes in five spacetime dimensions [21].

For my purpose it suffices to note that the degeneracy formula can be expressed in terms of an integral over an appropriate 3-cycle that involves an automorphic form $\Phi_{10}(\Omega)$,

$$
d(q, p)=\oint \mathrm{d} \Omega \frac{\mathrm{e}^{\mathrm{i} \pi\left(Q^{T} \Omega Q\right)}}{\Phi_{10}(\Omega)} .
$$

Here $\Omega$ denotes the period matrix for a genus-2 Riemann surface, which parametrizes the $\mathrm{Sp}(2) / \mathrm{U}(2)$ cosets; it can be written as a complex, symmetric, two-by-two matrix,

$$
\Omega=\left(\begin{array}{ll}
\rho & v \\
v & \sigma
\end{array}\right)
$$

In the exponent of the numerator of (25) the direct product of the period matrix with the invariant metric of the charge lattice is contracted with the charge vector comprising the 28 magnetic and 28 electric charges, so that $Q^{T} \Omega Q=\rho p^{2}+\sigma q^{2}+2 v p \cdot q$, where $p^{2}, q^{2}$ and $p \cdot q$ 
were defined previously in (21). The inverse of $\Phi_{10}$ has poles and the formula (25) picks up a corresponding residue whenever the 3-cycle encloses such a pole. However, the poles are located in the interior of the Siegel half-space and not just at its boundary and therefore the choice of the 3 -cycles is rather subtle.

In [18 the degeneracy formula was studied by means of a saddle-point approximation in the limit of large charges, but now retaining also the subleading terms. Remarkably enough the result is in precise agreement with the macroscopic results, i.e. (23) and (24), including the non-holomorphic terms. The equations (23) turn out to correspond to the equations that determine the location of the saddle-point, while (24) represents the value of the integrand in (25) taken at the saddle-point, including the results from integrating out the fluctuations about the saddle-point. This shows that the macroscopic entropy, defined by (24) as a function of the charges and the dilaton field, is in fact stationary under variations of the latter.

\section{The area law and elementary string states}

The area law is clearly violated in the presence of the subleading corrections, as is shown in the $N=2$ entropy formula (16). Of course, it depends on the theory in question and on the values for the charges, how sizable this violation is. A particularly interesting case emerges for black holes for which the leading contribution to the entropy and area vanish. In that case, the subleading terms become dominant and (17) shows that the area law is replaced by $\mathcal{S}_{\text {macro }}=\frac{1}{2} A(p, q)$, whereas the typical dependence on the charges proportional to the square root of a quartic polynomial is changed into the square root of a quadratic polynomial. It is easy to see how this can be accomplished for the heterotic black holes, namely, by suppressing all the charges $p^{0}, q_{1}, p^{2}, \ldots, p^{n}$ in (21) leaving $q^{2}$ as the only nonvanishing $T$ duality invariant charge combination. This is a remarkable result. In fact these states are precisely generated by perturbative heterotic string states arising from a compactification of six dimensions. In the supersymmetric right-moving sector they carry only momentum and winding and contain no oscillations, whereas in the left-moving sector oscillations are subject to the string matching condition. The oscillator number is then linearly related to $q^{2}$. These string states are 1/2-BPS states and correspond to electrically charged states (possibly upon a suitable electric/magnetic duality redefinition).

Precisely these perturbative states already received quite some attention in the past (for an early reference, see [22]). Because the higher-mass string BPS states are expected to be within their Schwarzschild radius, it was conjectured that they should have an interpretation as black holes. The idea that elementary particles, or string states, are behaving like black holes, is not new and has been around for quite some time. Hence, their calculable level

density, proportional to the exponent of $4 \pi \sqrt{\left|q^{2}\right| / 2}$, should be related to the entropy of these black holes [23]. On the other hand the corresponding black hole solutions were constructed in [24, 25] and it was found that their horizon area vanishes, in contrast with what one would expect on the basis of the area law. 
Of course, higer-order string corrections are expected to modify the situation at the horizon. One of the ways to incorporate their effect is to make use of the concept of a 'stretched' horizon, a surface close to the event horizon, whose location has to be chosen carefully in order that the calculations remain internally consistent. In this way it is possible to reconcile the non-zero level density with the vanishing of the classical horizon area 25, 26], although the precise proportionality factor in front of $\sqrt{\left|q^{2}\right| / 2}$ cannot be determined.

On the other hand, these corrections will undoubtedly be related to interactions of higher order in the curvature tensor whose effect can be studied in the context of the modified entropy formula (16) together with the attractor equations (after all, their derivation did not require any restriction on the value of the leading contributions to area and entropy). In fact, some of the results can be read off easily from the formulae (23) and (24). They show that the dilaton field becomes large and real (in contrast with the dyonic case, where the dilaton could remain finite and complex). Direct substitution yields,

$$
\begin{aligned}
S+\bar{S} & \approx \sqrt{\left|q^{2}\right| / 2}, \\
\mathcal{S}_{\text {macro }} & \approx 4 \pi \sqrt{\left|q^{2}\right| / 2}-6 \log \left|q^{2}\right|,
\end{aligned}
$$

where the logarithmic term is due to the non-holomorphic contribution. Because the dilaton is large in this case, all the exponentials in the Dedekind eta-function are suppressed and one is at weak string coupling. Consequently the formalism discussed in section 4 yields the expected results. In fact, without the non-holomorphic corrections the result for the entropy can be obtained directly from (10), upon taking $\hat{q}_{0}=q_{0}, C_{A B C}=0$ and $c_{2}{ }_{A} p^{A}=24 p^{1}$.

Not much attention was paid to this particular application of (16) until recently, when attention focused again on the electric black holes [27, this time primarily motivated by a reformulation of the black hole entropy (16) in terms of a mixed partition function [28]. I turn to the latter topic in the next section. The observation that (16) can nicely account for the discrepancies encountered in the classical description of the 1/2-BPS black holes was first made in 27, 29. Note also that, since the electric states correspond to perturbative heterotic string states, their degeneracy is known from string theory and given by

$$
d(q)=\oint \mathrm{d} \sigma \frac{\mathrm{e}^{\mathrm{i} \pi \sigma q^{2}}}{\eta^{24}(\sigma)} \approx \exp \left(4 \pi \sqrt{\left|q^{2}\right| / 2}-\frac{27}{4} \log \left|q^{2}\right|\right),
$$

where the integration contour encircles the point $\exp (2 \pi \mathrm{i} \sigma)=0$. The large- $\left|q^{2}\right|$ approximation is based on a standard saddle-point approximation. Obviously the leading term of (28) is in agreement with (27). However, the logarithmic corrections carry different coefficients.

At this point I should recall that the dyonic degeneracy formula (25) was proposed at the time [20] as an $S$-duality invariant extension of the electric degeneracy formula (28). While for the dyonic states the macroscopic results agree fully with the results obtained from a saddle-point approximation of (25), I conclude that the situation regarding the electric states is apparently more subtle. Recently, there have been quite a number of papers about the electric black holes, discussing the effect of the higher-derivative corrections in the effective action on the horizon behaviour and on more global aspects of the black hole solutions 30, 31, 32, 33, 34]; some of them also discuss the effect of the non-holomorphic corrections. 


\section{Black hole partition functions}

Recently the study of the BPS black hole entropy received a new impetous by the conjecture that there exists a black hole partition function based on a mixed ensemble, which is proportional to the square of the topological string partition function [28]. This conjecture was motivated by the observation that the entropy formula (16) can be rewritten as

$$
\mathcal{S}_{\text {macro }}(p, q)=\mathcal{F}(\phi, p)-\phi^{I} q_{I}, \quad \text { with } \quad q_{I}=\frac{\partial \mathcal{F}(\phi, p)}{\partial \phi^{I}} .
$$

Here the $Y^{I}$ are expressed in terms of the magnetic charges $p^{I}$ and (real) electrostatic potentials $\phi^{I}$ at the horizon,

$$
Y^{I}=\frac{\phi^{I}}{2 \pi}+\frac{\mathrm{i} p^{I}}{2}
$$

and the real function $\mathcal{F}(\phi, p)$ is defined by

$$
\mathcal{F}(\phi, p)=4 \pi \operatorname{Im}[F(Y, \Upsilon)]_{\Upsilon=-64}
$$

so that the magnetic attractor equations are already imposed and the electric ones correspond to the second equation in (29). The relation (29) constitutes precisely a Legendre transform and this fact motivated the introduction of a mixed black hole partition function of the form,

$$
\mathrm{e}^{\mathcal{F}(\phi, p)}=Z_{\mathrm{BH}}(\phi, p)=\sum_{\left\{q_{I}\right\}} d(q, p) \mathrm{e}^{q_{I} \phi^{I}},
$$

where $d(q, p)$ denotes the microscopic black hole degeneracies. This partition function is called a 'mixed' partition function, as it treats the electric and the magnetic charges differently: with respect to the magnetic charges one is dealing with a microcanonical ensemble, and with respect to the electric charges one has a canonical ensemble. Note that the left-hand side of (32) can be written as the modulus square of $\exp [-2 \pi \mathrm{i} F(Y, \Upsilon)]$, where the $Y^{I}$ are given by (30) and $\Upsilon=-64$. The holomorphic expression $\exp [-2 \pi \mathrm{i} F(Y, \Upsilon)]$ is actually related to the partition function for the topological string; the non-holomorphic corrections, which we suppressed here, are related to the so-called holomorphic anomaly [35].

The equation (32) implies that the black hole degeneracies can be expressed as a Laplace transform of the partition function $Z_{\mathrm{BH}}(\phi, p)$,

$$
d(q, p) \sim \int \prod_{I} \mathrm{~d} \phi^{I}\left|\mathrm{e}^{-2 \pi \mathrm{i} F(Y, \Upsilon)}\right|^{2} \mathrm{e}^{-q_{I} \phi^{I}} .
$$

where the $Y^{I}$ are still given by (30) and $\Upsilon=-64$. For large values of the $q_{I}$ the Laplace transform can be solved by a saddle-point approximation which leads to the exponent of the entropy $\mathcal{S}_{\text {macro }}(p, q)$ in accord with (29). It turns out that there exists a variety of these integral representations. They belong to a certain hierarchy and are generically related through a series of saddle-point approximations [36.

These integrals are, however, not properly defined. Leaving the question of convergence aside, a crucial issue concerns electric/magnetic duality. The attractor equations (12) and 
the expression for the macroscopic entropy (16) are manifestly consistent with this duality. Therefore the expression for the entropy transforms as a scalar function and its expression in a dual description is simply obtained by applying the duality on the charges $p^{I}$ and $q_{I}$, i.e., $\mathcal{S}_{\text {macro }}^{\prime}\left(p^{\prime}, q^{\prime}\right)=\mathcal{S}_{\text {macro }}(p, q)$. This implies, in particular, that the entropy will be invariant under any subgroup of the electric/magnetic duality group that constitutes an invariance of the model in question. The same property holds presumably for the microscopic black hole degeneracies, $d(q, p)$. Indeed, this is nicely demonstrated for the heterotic black holes, where both the macroscopic and the microscopic description were invariant under $T$ - and $S$-duality. On the other hand, the mixed partition function and the functions $F(Y, \Upsilon)$ and $\mathcal{F}(\phi, p)$ do not transform as functions under electric/magnetic duality; this is already obvious from the fact that the $\left(p^{I}, \phi^{I}\right)$ do not transform simply under this duality, unlike $\left(q_{I}, p^{I}\right)$. Hence it is not surprising that a straighforward integration leads to results that do not respect the various symmetries (as was, for instance, noted in [34]). Clearly the integration requires an appropriate measure in order to yield invariant results. In the context of the aforementioned hierarchy, it turns out that it is possible to construct such a measure [36].

So far the integral representation based on (33) has mainly been studied for the (electric) $1 / 2$-BPS states (sometimes called 'small' black holes, as their horizon area vanishes at the classical level) [27, 33, 34]. It seems reasonable to expect that there exists a single integral formula that pertains to both electric and dyonic black holes. However, it turns out that there are crucial differences when evaluating the corresponding integrals. To illustrate some of these subtleties, let me postulate the following integral expression for heterotic black holes,

$$
d(p, q)=\int \frac{\mathrm{d}^{2} S}{(S+\bar{S})^{2}} \mathrm{e}^{\mathcal{S}_{\text {macro }}}
$$

where $\mathcal{S}_{\text {macro is }}$ given by (24). Because of the factor $(S+\bar{S})^{-2}$ the integral is invariant under $S$-duality and it is also invariant under $T$-duality. While the above expression is not completely unfounded, as we shall see, it cannot be entirely correct. For simplicity let me suppress the exponential terms in the Dedekind eta-function, so that

$$
\mathcal{S}_{\text {macro }}=-\pi\left[\frac{q^{2}-\mathrm{i} p \cdot q(S-\bar{S})+p^{2}|S|^{2}}{S+\bar{S}}\right]+2 \pi(S+\bar{S})-12 \log (S+\bar{S}),
$$

and substitute them into the integral (34). Note that in this approximation we have lost the manifest $S$-duality invariance.

For the electric case where $p^{2}$ and $p \cdot q$ vanish, the integrand depends only on the real part of the dilaton field. Because of the $S$-duality invariance of the exact integral (34), the integral over the imaginary part extends only over a finite interval (in other words, we can restrict the integration to a suitable fundamental domain), so the only nontrivial integral is the one over the real part of $S$. Denoting the latter by $\frac{1}{2} x$, the integral takes the form (up to a numerical factor),

$$
d(p, q) \propto \int \frac{\mathrm{d} x}{x^{14}} \exp \left[-\frac{\pi q^{2}}{x}+2 \pi x\right]
$$

which equals a modified Bessel function of a certain degree (which was already noted in [34]), whose asymptotic behaviour for large negative $q^{2}$ coincides precisely with the microscopic 
result (28). The asymptotic result follows straightforwardly from a saddle-point approximation of (36), which shows that the dilaton field at the saddle point is large and given by the first equation of (27). The semi-classical correction which is included in the saddle-point approximation, thus changes the logarithmic term $-6 \log \left|q^{2}\right|$ in the second equation of (27) into the term $-\frac{27}{4} \log \left|q^{2}\right|$ in (28). This is a gratifying result.

One can repeat the same exercise for the dyonic case. Then the integrand does depend on both the real and imaginary parts of $S$ and the integral over the imaginary part now takes the form of a Gaussian integral, which can be performed explicitly. Subsequently one is again left with an integral over $x$ (after a suitable rescaling of the integration variables) which reads as follows (suppressing again a numerical factor),

$$
d(p, q) \propto \int \frac{\mathrm{d} x}{x^{27 / 2}} \exp \left[-\frac{\pi\left[p^{2} q^{2}-(p \cdot q)^{2}\right]}{x}-\pi\left(\frac{1}{4}-\frac{2}{p^{2}}\right) x\right] .
$$

This result also takes the form of a modified Bessel function, but now of a different degree. The asymptotic behaviour follows from a saddle-point approximation, which leads to the entropy (20) modified by a logarithmic term. The coefficient of the logarithm is, however, not in agreement with (23) and (24), which, as I discussed in section [5, have been shown to follow from the formula (25) for the dyonic degeneracies, up to inverse powers of the charges 18. Although (34) thus seems to lead to a desirable result in the electric case, it fails to do so in the dyonic case. This does not yet pose a problem as (34) was only introduced as an example to illustrate typical features of these integral representations. In certain cases this particular formula does play a role at an intermediate stage of a more complete derivation, but the latter reveals that it is actually the electric case where most of the subtleties arise, while the dyonic case is much more generic, at least when one adopts an appropriate measure for the integral [36].

In spite of these open questions it is clear that surprising progress has been made in the understanding of black hole entropy. The intriguing connection with topological string theory and the interplay with supergravity and general relativity open up new perspectives for research. I expect that this will remain an exciting subject for study in the years to come.

Most of my own work on the topic of this talk has been in collaboration with Gabriel Lopes Cardoso, Jürg Käppeli and Thomas Mohaupt whom I thank for valuable comments on the text. It is a pleasure to acknowledge the kind hospitality of the Yukawa Institute (Kyoto University) where most of this contribution was prepared. This work is partly supported by EU contract MRTN-CT-2004-005104.

\section{References}

[1] J.M. Bardeen, B. Carter and S.W. Hawking, "The four laws of black hole mechanics", Commun. Math. Phys. 31 (1973) 161.

[2] S.W. Hawking, "Particle creation by black holes", Comm. Math. Phys. 43 (1975) 199. 
[3] J.D. Bekenstein, "Black holes and entropy", Phys. Rev. D7 (1973) 2333; "Generalized second law of thermodynamics in black-hole physics", Phys. Rev. D9 (1974) 3292.

[4] A. Strominger and C. Vafa, "Microscopic origin of the Bekenstein-Hawking entropy", Phys. Lett. B379 (1996) 99-104, hep-th/9601029.

[5] J.M. Maldacena, A. Strominger, and E. Witten, "Black hole entropy in M-theory", JHEP 12 (1997) 002, hep-th/9711053

[6] C. Vafa, "Black holes and Calabi-Yau threefolds", Adv. Theor. Math. Phys. 2 (1998) 207-218, hep-th/9711067.

[7] R. Minasian, G. Moore and D. Tsimpis, "Calabi-Yau black holes and $(0,4)$ sigma models", Commun. Math. Phys. 209 (2000) 325, hep-th/09904217.

[8] S. Ferrara, R. Kallosh, and A. Strominger, " $N=2$ extremal black holes", Phys. Rev. D52 (1995) 5412, hep-th/9508072.

[9] A. Strominger, "Macroscopic entropy of $N=2$ extremal black holes", Phys. Lett. B383 (1996) 39, hep-th/9602111.

[10] S. Ferrara and R. Kallosh, "Supersymmetry and attractors", Phys. Rev. D54 (1996) 1514, hep-th/9602136

[11] G.L. Cardoso, B. de Wit, J. Käppeli, and T. Mohaupt, "Stationary BPS solutions in $N=2$ supergravity with $R^{2}$-interactions", JHEP 12 (2000) 019, hep-th/0009234.

[12] B. de Wit, J.-W. van Holten and A. Van Proeyen, "Structure of $N=2$ supergravity", Nucl. Phys. B184 (1981) 77; B. de Wit, P.G. Lauwers, R. Philippe, S.Q. Su and A. Van Proeyen, "Gauge and mater fields coupled to $N=2$ supergravity", Phys. Lett. B134 (1984) 37; B. de Wit and A. Van Proeyen, "Potentials and symmetries of general gauged $N=2$ supergravity-Yang-Mills models", Nucl. Phys. B245 (1984) 89.

[13] K. Behrndt, G.L. Cardoso, B. de Wit B, D. Lüst, T. Mohaupt and W.A. Sabra, "Higher order black hole solutions in $N=2$ supergravity and Calabi-Yau string backgrounds", Phys. Lett. B429 (1998) 289, hep-th/9801081

[14] R.M. Wald, "Black hole entropy entropy in the Noether charge", Phys. Rev. D48 (1993) 3427, gr-qc/9307038 V. Iyer and R.M. Wald, "Some properties of Noether charge and a proposal for dynamical black hole entropy", Phys. Rev. D50 (1994) 846, gr-qc/9403028, T. Jacobson, G. Kang and R.C. Myers, "Black hole entropy in higher curvature gravity", Phys. Rev. D49 (1994) 6587, gr-qc/9312023

[15] G.L. Cardoso, B. de Wit and T. Mohaupt, "Deviations from the area law for supersymmetric black holes", Fortsch. Phys. 48 (2000) 49, hep-th/9904005.

[16] G.L. Cardoso, B. de Wit and T. Mohaupt, "Corrections to macroscopic supersymmetric black-hole entropy", Phys. Lett. B451 (1999) 309-316, hep-th/9812082. 
[17] G.L. Cardoso, B. de Wit and T. Mohaupt, "Macroscopic entropy formulae and non-holomorphic corrections for supersymmetric black holes", Nucl. Phys. B567 (2000) 87, hep-th/9906094

[18] G.L. Cardoso, B. de Wit, J. Käppeli and T. Mohaupt, "Asymptotic degeneracy of dyonic $N=4$ string states and black hole entropy", JHEP 12 (2004) 075, hep-th/0412287

[19] J.A. Harvey and G.W. Moore, "Fivebrane instantons and $R^{2}$ couplings in $\mathrm{N}=4$ string theory", Phys. Rev. D57 (1998) 2323-2328, hep-th/9610237.

[20] R. Dijkgraaf, E. Verlinde, and H. Verlinde, "Counting dyons in N = 4 string theory", Nucl. Phys. B484 (1997) 543-561, hep-th/9607026.

[21] S. Shih, A. Strominger and X. Yin, "Recounting dyons in N-4 string theory", hep-th/0505094.

[22] A. Dabholkar and J.A. Harvey, "Nonrenormalization of the superstring tension", Phys. Rev. Lett. 63 (1989) 478.

[23] J.G. Russo and L. Susskind, "Asymptotic level density in heterotic string theory and rotating black holes", Nucl. Phys. B437 (1995) 611, hep-th/9405117.

[24] A. Sen, "Black hole solutions in heterotic string theory on a torus", Nucl. Phys. B440 (1995) 421, hep-th/9411187.

[25] A. Sen, "Extremal black holes and elementary string states", Mod. Phys. Lett. A10 (1995) 2081, hep-th/9504147.

[26] A. Peet, "Entropy and supersymmetry of $D$ dimensional extremal electric black holes versus string states", Nucl. Phys. B456 (1995) 732, hep-th/9506200

[27] A. Dabholkar, "Exact counting of black hole microstates", hep-th/0409148

[28] H. Ooguri, A. Strominger and C. Vafa, "Black hole attractors and the topological string", Phys. Rev. D70 (2004) 106007, hep-th/0405146.

[29] A. Dabholkar, R. Kallosh and A. Maloney, "A stringy cloak for a classical singularity", JHEP 0412 (2004) 059, hep-th/0410076.

[30] A. Sen, "How does a fundamental string stretch its horizon?", hep-th/0411255.

[31] V. Hubeny, A. Maloney and M. Rangamani, "String-corrected black holes", JHEP 0505 (2005) 035, hep-th/0411272.

[32] D. Bak, S. Kim and S.-J. Rey, "Exactly soluble BPS black holes in higher curvature $N=2$ supergravity", hep-th/05010011. 
[33] A. Sen, "Black holes, elementary strings and holomorphic anomaly", hep-th/0502126. 'Black holes and the spectrum of half-BPS states in $N=4$ supersymmetric string theory", hep-th/0504005.

[34] A. Dabholkar, F. Denef, G.W. Moore and B. Pioline, "Exact and asymptotic degeneracies of small black holes", hep-th/0502157 "Precision counting of small black holes", hep-th/0507014.

[35] M. Berhadsky, S. Cecotti, H. Ooguri and C. Vafa, "Kodeira-Spencer theory of gravity and exact results for quantum string amplitudes", Commun. Math. Phys. 165 (1994) 311 , hep-th/9309140.

[36] G.L. Cardoso, B. de Wit, J. Käppeli and T. Mohaupt, to appear; preliminary results were presented at the 'Workshop on gravitational aspects of string theory' of the Fields Institute (Toronto, May 2005) and at 'Strings 2005' (Toronto, July 2005). 